\title{
BMJ Open Diagnostic performance of image technique based transurethral resection for non-muscle invasive bladder cancer: systematic review and diagnostic meta- analysis
}

\author{
Changhao Chen, ${ }^{1}$ Hao Huang, ${ }^{1}$ Yue Zhao, ${ }^{2}$ Hao Liu, ${ }^{3}$ Richard Sylvester, ${ }^{4}$ \\ Tianxin Lin, ${ }^{1}$ Jian Huang ${ }^{1}$
}

To cite: Chen C, Huang $\mathrm{H}$, Zhao Y, et al. Diagnostic performance of image technique based transurethral resection for non-muscle invasive bladder cancer: systematic review and diagnostic meta-analysis. BMJ Open 2019;9:e028173. doi:10.1136/ bmjopen-2018-028173

- Prepublication history and additional material for this paper are available online. To view these files, please visit the journal online (http://dx.doi. org/10.1136/bmjopen-2018028173).

Received 25 November 2018 Revised 20 September 2019 Accepted 26 September 2019

\section{Check for updates}

(c) Author(s) (or their employer(s)) 2019. Re-use permitted under CC BY-NC. No commercial re-use. See rights and permissions. Published by BMJ.

${ }^{1}$ Department of Urology, Sun Yat-Sen Memorial Hospital, Guangzhou, China

${ }^{2}$ Department of Gastroenterology, The First Affiliated Hospital of Sun Yat-Sen University, Guangzhou, China ${ }^{3}$ Department of Urology, Chengdu Fifth People's Hospital, Chengdu, China

${ }^{4}$ European Association of Urology Guidelines Office, Arnhem, Brussels

Correspondence to Professor Jian Huang; cch1988@163.com

\section{ABSTRACT}

Objective To explore the diagnostic performance of image technique based transurethral resection for bladder cancer, with white light-guided cystoscopy (WLC) as the reference standard.

Design Systematic review and meta-analysis.

Data sources PubMed/MEDLINE, Web of Science, the Cochrane Library, Central Register of Controlled Trials and Embase from inception to 31 March 2018.

Methods Included studies reported the diagnostic performance of photodynamic diagnosis (PDD) with 5-aminolevulinic acid (5-ALA), PDD with hexaminolevulinic acid (HAL) or narrow band imaging (NBI), with WLC as the reference standard at the patient or lesion level. The studies' risk of bias (RoB) was assessed using Quality Assessment of Diagnostic Studies-2. Data were pooled using a random effect diagnostic meta-analysis, and subgroup analyses were performed.

Results Twenty-six studies comprising a total of 3979 patients were included in this diagnostic meta-analysis. Pooled sensitivity (SSY), specificity (SPY), diagnostic OR (DOR) and area under the receiver operating characteristic curve (AUROC) values were calculated per group for NBI, HAL and 5-ALA at the lesion or patient level. NBI showed significant diagnostic superiority compared with WLC at the lesion level (SSY 0.94, 95\% Cl 0.82 to 0.98; SPY $0.79,95 \% \mathrm{Cl} 0.73$ to 0.85 ; DOR $40.09,95 \% \mathrm{Cl} 20.08$ to 80.01; AUROC $0.88,95 \% \mathrm{Cl} 0.85$ to 0.91 ). NBI presented the highest DOR $(358.71,95 \% \mathrm{Cl} 44.50$ to 2891.71$)$ in the patient level. Subgroup analyses were performed on studies with low to moderate RoB and at least 100 patients at the lesion level. These results were consistent with those of the overall analysis.

Conclusions Pooled data indicated that image technique based transurethral resection (NBI, HAL and 5-ALA) showed diagnostic superiority compared with WLC. Moreover, NBI is potentially the most promising diagnostic intervention, showing the best diagnostic performance outcomes. Further prognostic outcomes of novel imaging technologies compared with those WLC should be explored in addition to current diagnostic performance analysis.

\section{Strengths and limitations of this study}

- This is the first systematic review and diagnostic meta-analysis exploring diagnostic accuracy of image technique based transurethral resection compared with white light-guided cystoscopy.

- Our study includes the stringent methodology used to synthesise the evidence obtained, such as adhering to Preferred Reporting Items for Systematic Reviews and Meta-Analyses guidelines, using standardised definitions of diagnostic performance analysis and applying Quality Assessment of Diagnostic Studies-2 tool for risk of bias assessment.

- Most included studies had a low or moderate risk of bias. All studies clearly reported methodology for the index test and reference standard and were not considered a significant source of potential bias.

- The further sensitivity analysis was based on relatively few studies, but we used random effect models to compensate for clinical and methodological diversity among studies.

- The lack of data on important clinical variables, such as grade and stage of disease, primary versus (vs) recurrent disease and intravesical instillation settings, may introduce clinical heterogeneity and prevent further sensitivity analyses. We attempted to minimise biases by standardising data extraction and performing several sensitivity analyses.

\section{INTRODUCTION}

Bladder cancer is a widespread malignancy with an estimated 80470 newly diagnosed cases and 17670 deaths in USA in 2019 , among which about $75 \%$ of patients presented with non-muscle invasive bladder cancer (NMIBC). ${ }^{12}$ Currently, white light cystoscopy (WLC) is the gold standard technique to detect bladder cancer, despite having an unsatisfactory accuracy to detect disease. The detection reliability for smaller tumours or carcinoma in situ (CIS) is poor, leading 
to markedly high recurrence, with up to $30 \%$ of patients having a tumour identified at the first-check cystoscopy at 3 months and $50 \%$ of patients developing tumours within 12 months. ${ }^{34}$ Thus, different optical imaging techniques have emerged as an adjunct to WLC to improve the visualisation of tumours via contrast enhancement.

Photodynamic diagnosis (PDD) is performed using blue-violet (380-440 nm) light with intravesical instillation of 5-aminolevulinic acid (5-ALA) or hexaminolevulinic acid (HAL). The effect of 5-ALA-induced fluorescence on tumour detection in the urinary bladder has identified it as an efficient method to map the entire mucosa to detect urothelial tumours and flat CIS lesions. ${ }^{5-7}$ HAL, the lipophilic hexylester of 5-ALA, has been commercially available since 2006 and has been established as the preferred intravesical agent to detect NMIBC. However, intravesical inflammation leads to decreased specificity, and preoperative procedures are technically complex and costly.

Narrow band imaging (NBI) is a new image processing modality that filters white light down to two narrow band widths of 415 and $540 \mathrm{~nm}$, with advantage of avoiding the need for intravesical contrast administration. ${ }^{8}$ Haemoglobin absorbs these wavelengths preferentially, resulting in dark neovascularised bladder cancer appearing very different from the light background of the normal mucosa. The superior diagnostic performance of NBI compared with WLC has been confirmed in several studies. ${ }^{9-11}$ Overall, NBI led to a $9.9 \%$ increase in the detection rate at the patient level and a $19.2 \%$ increase in lesion detection in a recent meta-analysis, while subgroup analysis showed that NBI was associated with a $53 \%$ reduction in the recurrence rate at 3 months and $19 \%$ at 12 months compared with those of WLC. ${ }^{12}$ Noticeably, NBI might be associated with increased false-positives, especially for patients with prior intravesical instillations. ${ }^{13}$

As a standard procedure, cystoscopy is performed using white light. However, the use of white light can lead to lesions that are present but not visible being missed. New imaging techniques could improve cancer detection compared with WLC; however, some studies showed that new imaging techniques might produce higher false positive rate (FPRs) than WLC. ${ }^{13-15}$ In addition, their complex procedures and costs restrict their wider application. ${ }^{16}$ Therefore, it is still uncertain which technique could better improve the diagnostic accuracy of bladder cancer detection. The present study aimed to perform a systematic review and meta-analysis to assess the diagnostic performance of PDD using 5-ALA, PDD using HAL and NBI against the current reference standard of WLC for NMIBC.

\section{METHODS}

The diagnostic meta-analysis was conducted based on the Meta-analysis of Observational Studies in Epidemiology statement. ${ }^{17}$ All included studies were observational studies. When an included primary study did not match the Standards for Reporting of Diagnostic Accuracy statement, we gathered the information by contacting the authors. ${ }^{18}$

\section{Literature search}

Studies reporting the diagnostic performance of PDD with 5-ALA, PDD with HAL or NBI, with WLC as reference standard, were retrieved from multiple databases including PubMed/MEDLINE, Web of Science, the Cochrane Library, Central Register of Controlled Trials and Embase up to 31 March 2018. The following Medical Subject Headings (MeSH) free and combined terms, which were adjusted for the different databases terms, were used: 'photodynamic diagnosis, PDD, hexaminolevulinate, HAL, 5-aminolevulinate acid, 5-ALA, narrow imaging, NBI, WLC, urothelial cell carcinoma of bladder, transitional cell carcinoma, bladder cancer, bladder tumour, and BCa'. The full search strategy is shown in the appendix (online supplementary material). The review was performed according to Preferred Reporting Items for Systematic Reviews ${ }^{19}$ and Standards for Reporting Diagnostic Accuracy Studies. ${ }^{20}$ The search was restricted to English-language publications. At least two reviewers (CC and $\mathrm{HH})$ screened all the abstracts and full-text articles independently. Disagreement was resolved by consultation with an independent arbiter $(\mathrm{JH})$.

\section{Inclusion and exclusion criteria}

The inclusion criteria were as follows: (1) population: patients diagnosed with primary NMIBC or patients previously diagnosed with NMIBC (recurrent tumours); (2) reference standard: WLC must be provided as the reference standard for all patients, and the diagnosis of NMIBC was confirmed by histopathological examination; (3) studies reported data of intrapatient comparison; and (4) when two or more studies provided data from the same institution during an overlapping time period, only the updated data were included in this study.

Articles were excluded if the full-text article was not written in English. Abstracts, conference articles, historical overviews, case studies, reviews and meta-analyses were not considered. Studies that failed to report on sensitivity and specificity data or both in comparison with WLC were excluded. For missing or unclear data, we contacted the authors to obtain more information.

\section{Patient and public involvement}

Patients and the public were not involved in this research.

\section{Study quality}

The Quality Assessment of Diagnostic Studies-2 (QUADAS-2) $^{21}$ and the Strength of Recommendation Taxonomy numerical scale were applied to the included studies. ${ }^{22}$ Both checklists were performed independently by two authors ( $\mathrm{YZ}$ and $\mathrm{CC}$ ); disagreement was resolved by consultation with an independent arbiter $(\mathrm{JH})$. The 'low risk of bias (RoB)' was defined as at least three domains with 'low' in both categories and without any domains evaluated as 'high' in either category; 'moderate RoB' was defined as at least two domains with 'low' in 


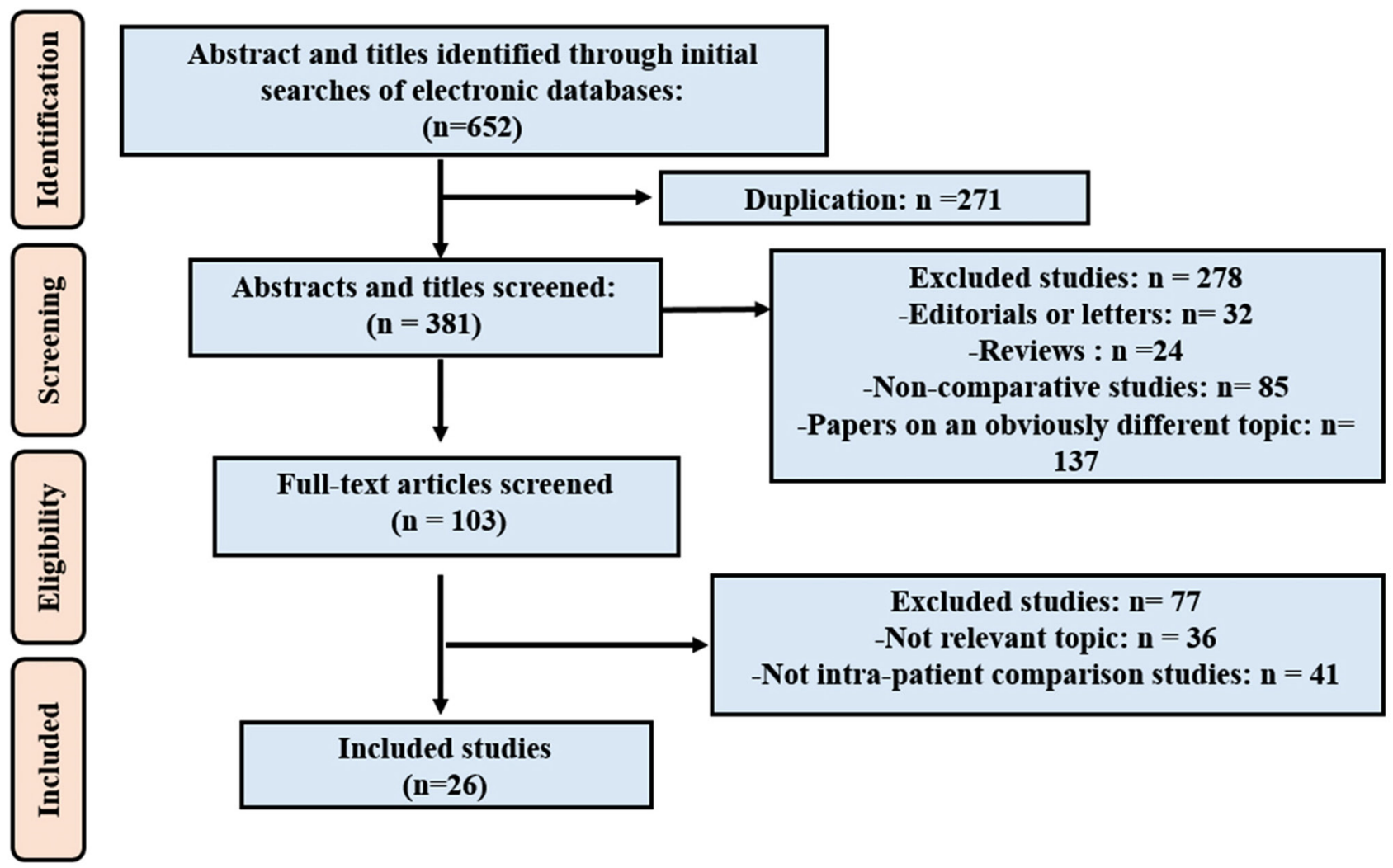

Figure 1 The PRISMA flow chart of included studies in DTA analysis. DTA, diagnostic test accuracy; PRISMA, Preferred Reporting Items for Systematic Reviews and Meta-Analyses.

both categories and without any domain scoring 'high' in either category; "High RoB" was defined as only one domains with "low" in both categories and have at least one domain evaluated as"high" in either category.

\section{Data extraction}

The following data were extracted from the selected studies: (1) study characteristics (first author, study design, number of patients and follow-up); (2) intervention characteristics (index tests, duration of follow-up, schedule and nature of WLC); (3) patient characteristics (age, sex, NMIBC patients and tumour lesions); (4) diagnostic performance measures (sensitivity (SSY), specificity (SPY), negative predictive value (NPV), positive predictive value (PPV), FPR and false negative rate (FNR)). Data were extracted from each study at the lesion or patient level to assess 5-ALA, HAL and NBI as the index test using WLC as the reference standard, with positive or negative disease being determined using histopathological examination.

The primary outcomes of SSY, SPY, NPV, PPV, FPR and FNR for individual studies were calculated using the following standard definitions. SSY was defined as the proportion of positive patients or lesions with index tests in all cases of WLC-positive findings. SPY was defined as the proportion of negative patients or lesions with index tests in all cases of WLC-negative findings. NPV was defined as the proportion of true negatives findings (both negative in index tests and WLC) in all index test-negative cases or lesions. PPV was defined as the proportion of true positives findings (both positive in index tests and WLC) in all index test-positive cases or lesions. FNR was defined as the proportion of index test-negative findings in all cases of WLC-positive cases or lesions. FPR was defined as the proportion of index test-positive findings in all cases of WLC-negative cases or lesions.

\section{Statistical analysis}

Separate meta-analyses were performed for the currently new technology-assisted cystoscopy in patients with NMIBC to best summarise the totality of the available evidence. The diagnostic meta-analysis was performed using Stata V.13.0 with the metan and midas commands. A two-sided $p$ value of less than 0.05 was considered significant. In this study, a random effect model was applied to quantify the pooled sensitivity, specificity, diagnostic OR (DOR) and area under the receiver operating characteristic curve (AUROC), with 95\% CIs of the compared end points. DOR reflects the diagnostic performance of a new imaging technique to detect lesions. A DOR value of 1 indicates that the test has no discriminative power; the higher the DOR value, the better the diagnostic performance of the new imaging technique. The AUROC is an overall summary measure index of the diagnostic accuracy. A perfect test will have an AUROC close to 1, and a poor test will have an AUROC close to $0.5 .^{23} \mathrm{We}$ plotted the sensitivities and specificities in the summary receiver operating curve (SROC) space, using different 
Table 1 Summary of the characteristics of the included studies

\begin{tabular}{|c|c|c|c|c|c|c|c|c|}
\hline Study & $\begin{array}{l}\text { Institution } \\
\text { no. }\end{array}$ & Patients & $\begin{array}{l}\text { Index } \\
\text { test }\end{array}$ & Period & $\begin{array}{l}\text { Age, mean } \\
\text { (range) }\end{array}$ & $\begin{array}{l}\text { Men } \\
(\%)\end{array}$ & $\begin{array}{l}\text { NMIBC } \\
(\%)\end{array}$ & $\begin{array}{l}\text { Tumour } \\
\text { lesions (n) }\end{array}$ \\
\hline Shadpour et al ${ }^{31}$ & Unicentre & 50 & NBI & 2012-2013 & $63.86 \pm 10.05$ & $34(68.0)$ & 100 & 95 \\
\hline Kobatake et al ${ }^{36}$ & Unicentre & 135 & $\mathrm{NBI}$ & 2010-2014 & 75 & $\begin{array}{l}110 \\
(81.5)\end{array}$ & 100 & 120 \\
\hline Ye et $a l^{11}$ & Multicentre & 384 & NBI & NR & $61(21-79)$ & $\begin{array}{l}267 \\
(69.5)\end{array}$ & 100 & 167 \\
\hline Shen et $a l^{30}$ & Unicentre & 78 & $\mathrm{NBI}$ & 2009-2010 & $68(33-75)$ & $62(79.5)$ & 100 & 211 \\
\hline Zhu et $a l^{26}$ & Unicentre & 12 & $\mathrm{NBI}$ & 2009-2010 & $57(28-73)$ & $9(75.0)$ & 100 & 9 \\
\hline Tatsugami et $\left.a\right|^{28}$ & Unicentre & 104 & NBI & 2007-2009 & $70.6(38-90)$ & $88(84.6)$ & NR & 110 \\
\hline Cauberg et $\mathrm{al}^{47}$ & Multicentre & 95 & NBI & 2007-2009 & 70.6 (38.1-90.2) & $70(73.7)$ & NR & 226 \\
\hline Lapini et al ${ }^{35}$ & Multicentre & 96 & HAL & 2010-2011 & NR & $80(83.3)$ & NR & 108 \\
\hline Burgués et $a l^{48}$ & Multicentre & 305 & $\mathrm{HAL}$ & 2006-2009 & $66.9(39-93)$ & $\begin{array}{l}270 \\
(88.5)\end{array}$ & 100 & 600 \\
\hline Ray et $a l^{15}$ & Unicentre & 27 & HAL & 2005-2006 & $70(49-82)$ & $21(77.8)$ & 100 & NR \\
\hline Schmidbauer et $\left.a\right|^{32}$ & Unicentre & 66 & $\mathrm{HAL}$ & NR & $63(38-84)$ & $49(74.2)$ & 93.1 & NR \\
\hline Geavlete et $a l^{40}$ & Unicentre & 128 & $\mathrm{HAL}$ & $2007-2008$ & $65(36-81)$ & NR & 92.2 & NR \\
\hline Fradet et al ${ }^{41}$ & Multicentre & 298 & $\mathrm{HAL}$ & $\mathrm{NR}$ & $67 \pm 11$ & $\begin{array}{l}223 \\
(74.8)\end{array}$ & 100 & 113 \\
\hline Jichlinski et a/ ${ }^{37}$ & Multicentre & 52 & HAL & 2000-2001 & $72 \pm 12$ & $38(73.1)$ & 100 & 143 \\
\hline Filbeck et al ${ }^{42}$ & Unicentre & 123 & 5-ALA & 1997 & $64.5(28-86)$ & NR & 91.9 & 124 \\
\hline Riedl et $a l^{33}$ & Unicentre & 52 & 5-ALA & NR & $44-79$ & NR & 100 & 123 \\
\hline D'Hallewin et al/6 & Unicentre & 16 & 5-ALA & NR & NR & NR & 100 & 50 \\
\hline
\end{tabular}

5-ALA, 5-aminolaevulinic acid; HAL, hexylaminolevulinate; NBI, narrow band imaging;NMIBC, non-muscle invasive bladder cancer; NR, not reported; NT, new technology.

symbols for different imaging techniques, and used RevMan 5.2 software to build hierarchical SROC curves for each imaging technique. We also formulated forest plots of the summary measures of accuracy and examined the heterogeneity of the summary measures of sensitivity and specificity. The publication bias was assessed using Deeks' funnel plot, and statistical significance was determined using Deeks' asymmetry test. ${ }^{24}{ }^{25}$ To explore the effect of heterogeneity on the results, subgroup analyses were planned based on disease grade (low grade vs high grade), stage (pTa vs pT1), setting (primary vs recurrent tumours), number of participants (studies with $n>100$ patients only) and on studies with low to moderate RoB.

\section{RESULTS}

\section{Search and study selection}

The flow diagram summarising the literature screening and inclusion process is presented in figure 1 . Of the 652 potentially relevant articles identified in the database search, 271 studies were excluded as duplicates. We excluded 278 studies when screening the titles and abstracts: 32 were editorials or letters, 24 were reviews or meeting abstracts, 85 were non-comparative studies and 137 papers concerned an obviously different topic. During the screening of 103 full-text articles, 36 studies were excluded for not being relevant to this review, and another 41 studies were excluded for not having 


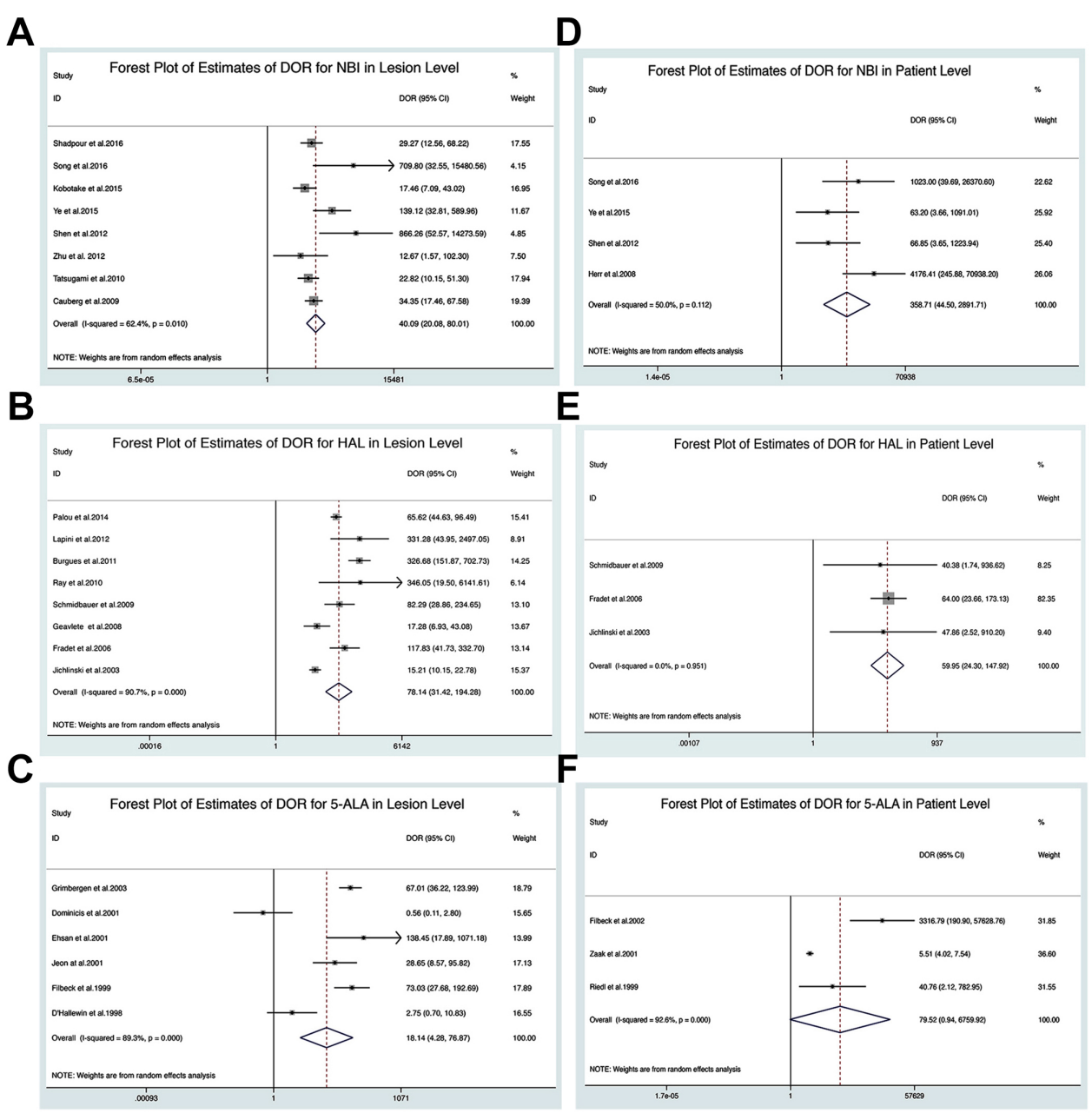

Figure 2 The forest plot of estimates of DOR for NBI (A), HAL (C) and 5-ALA (E) in lesion level and estimates of DOR for NBI (B), HAL (D) and 5-ALA (F) in patient level. 5-ALA, 5-aminolevulinic acid; DOR, diagnostic OR; HAL, hexaminolevulinic acid; NBI, narrow band imaging.

within-patient comparisons. Finally, 26 studies $^{5} 1115$ 26-48 were included in the diagnostic meta-analysis.

\section{Study demographics}

The characteristics of the 26 studies included in this meta-analysis are summarised in table 1 . The studies were published from 1994 to 2016 with sample sizes ranging from 12 to 605 patients. The mean or median age and male/female ratio showed no significant differences among included studies. In nine studies, the NBI diagnostic intervention was applied, while 5-ALA-based PDD was conducted in nine studies and HAL-based PDD in eight studies. Most of the enrolled patients in the included studies suffered from NMIBC.

\section{Lesion-level analysis}

All studies used non-standardised definitions to calculate their diagnostic outcomes, in which case the results of the included studies were recalculated using standard definitions from the raw data provided (online supplementary table 1). The diagnostic meta-analysis results are presented using lesion-level and patient-level analyses. Based on the lesion level, the forest plot of estimates of the DOR for NBI, HAL and 5-ALA compared with WLC are shown in figure 2. The pooled DOR values for NBI, HAL and 5-ALA were 40.09 (95\% CI 20.08 to 80.01 , figure $2 \mathrm{~A}$ ), 78.14 (95\% CI 31.42 to 194.28 , figure $2 \mathrm{~B}$ ) and 18.14 (95\% CI 4.28 to 76.87 , figure $2 \mathrm{C}$ ), respectively. The SROC curves for NBI, HAL and 5-ALA are shown in figure 3A. The AUROC values of NBI, HAL and 5-ALA were 0.88 (95\% CI 0.85 to 0.91$), 0.94(95 \%$ CI 0.92 to 0.96$)$, and 0.82 (95\% CI 0.79 to 0.85$)$, respectively. Importantly, the results of the SSY and SPY for each intervention are shown in online supplementary figures 1-3. The pooled estimates for the SSY data for NBI, HAL and 5-ALA were 0.94 (95\% CI 0.82 to 0.98 , online supplementary figure 1A), 0.95 (95\% CI 0.91 to 0.98, online supplementary figure 2A) and 0.90 (95\% CI 0.71 to 0.97 , online supplementary figure $3 \mathrm{~A}$ ), respectively, whereas the SPY values for NBI, HAL and 5-ALA were $0.79(95 \%$ CI 0.73 to 0.85 , online supplementary figure $1 \mathrm{~B}), 0.81$ (95\% CI 0.74 to 0.87 , online supplementary figure $2 \mathrm{~B}$ ) and 0.69 (95\% CI 0.57 to 0.79 , online supplementary figure $3 \mathrm{~B}$ ), respectively, presenting superiority compared with WLC. The DOR and AUROC values of NBI, HAL and 5-ALA indicated excellent diagnostic performance. 

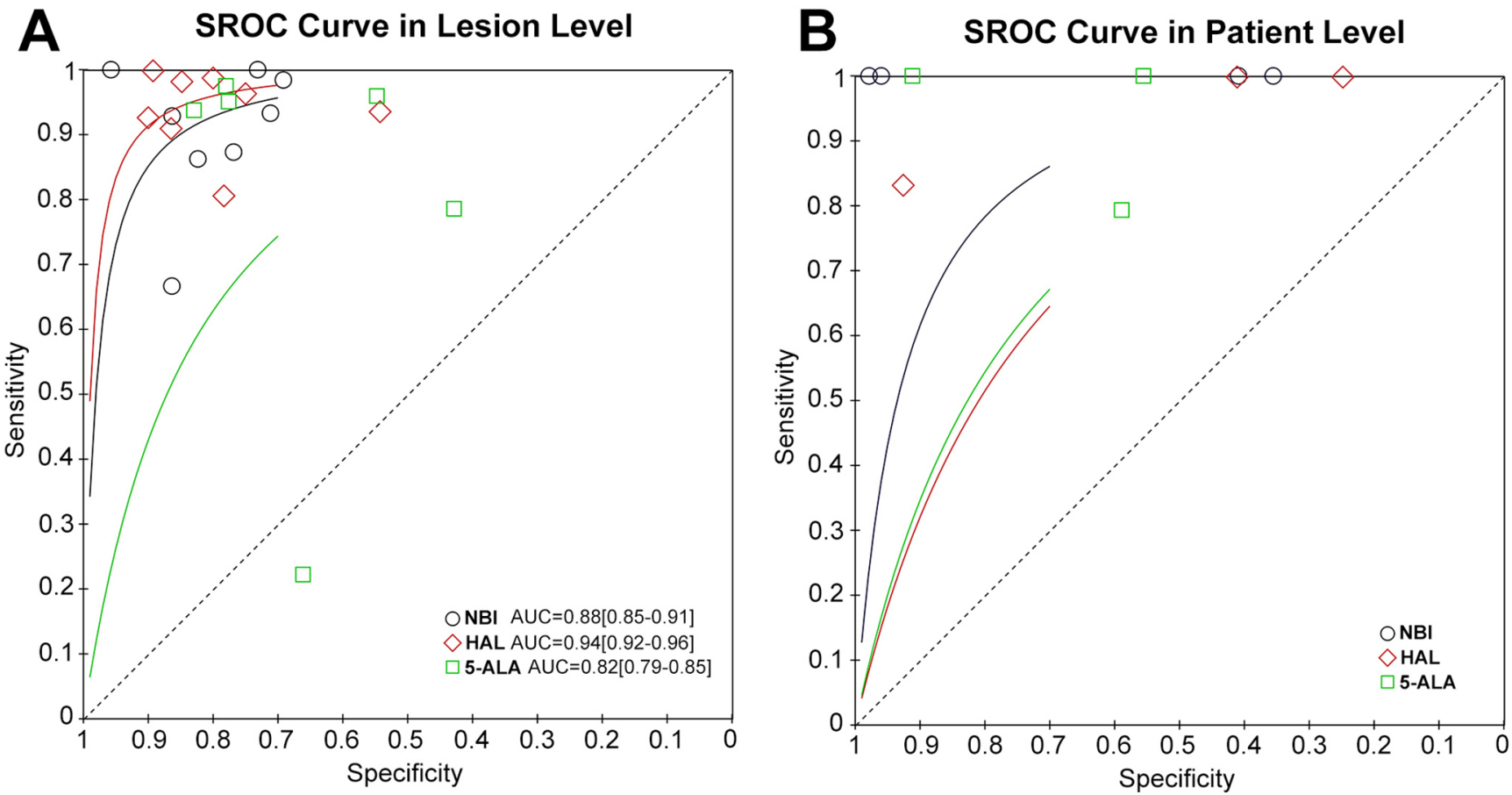

Figure 3 The SROC curve for NBI, HAL and 5-ALA diagnosing NMIBC in lesion level (A) and patient level (B). 5-ALA, 5-aminolevulinic acid; HAL, hexaminolevulinic acid; NBI, narrow band imaging; NMIBC, non-muscle invasive bladder cancer; SROC, summary receiver operating curve.

\section{Patient-level analysis}

For the patient level analysis, the AUROC, SSY and SPY could not be calculated because few studies included these data. figure 2 shows the forest plots of DOR for NBI, HAL and 5-ALA. NBI showed the highest DOR. The DOR values for NBI and HAL were 358.71 (95\% CI 44.50 to 2891.71, figure 2D) and 59.95 (95\% CI 24.30 to 147.92 , figure $2 \mathrm{E}$ ), respectively, presenting a better performance compared with that of WLC. The SROC curves for NBI, HAL and 5-ALA are shown in figure 3B. However, the DOR value for 5-ALA was 79.52 (95\% CI 0.94 to 6759.92 , figure 2 F) and did not show a statistical difference.

\section{Subgroup analysis}

Subgroup analyses were performed on studies with low to moderate RoB and at least 100 patients at the lesion level. The diagnostic performance results for studies with low to moderate RoB and at least 100 patients are shown in online supplementary table 2 . The forest plot of the estimates of the pooled DOR for NBI, HAL and 5-ALA with low to moderate RoB are shown in online supplementary figure 4 , while the forest plot of the estimates of the pooled DOR for NBI, HAL and 5-ALA with at least 100 patients are shown in online supplementary figure 5 . These results were consistent with those obtained in the overall analysis.

\section{RoB of the included studies}

The comparison-adjusted funnel plots of the diagnostic meta-analysis were not suggestive of any publication bias, as shown in figure 4. The QUADAS-2 tool was applied for RoB assessment of the included studies in our meta-analysis (online supplementary figure 6). Overall, all studies reported methodology for the index test and reference standard clearly, without a significant source of potential bias. Among them 69\% (18/26) of the studies were presented as low or unclear RoB across most domains. The RoB in the patient selection in three studies was deemed high because of the absence of the consecutive inclusion of patients; four studies were deemed to have a high RoB for flow and timing.

\section{DISCUSSION}

Our systematic review indicated that the pooled diagnostic performance of NBI, HAL or 5-ALA showed excellent efficacy compared with WLC. NBI could potentially be the most promising diagnostic intervention for patients with NMIBC, with advantages in terms of simplicity, cost, and reliability. In the present study, we have summarised the diagnostic performance of new technique-assisted cystoscopy strategies for NMIBC. A diagnostic meta-analysis was further undertaken to estimate the diagnostic performance of NBI, HAL and 5-ALA compared with that of WLC. WLC has been the standard method to detect urothelial cell carcinoma of the bladder. However, the sensitivity of WLC is unsatisfactory, and it can miss small 'satellite' tumours or CIS. Thus, new imaging techniques (PDD and NBI) have been introduced to enhance bladder cancer visualisation to improve diagnostic accuracy and the thoroughness of resection. Several studies have demonstrated that the new imaging techniques showed superior diagnostic performance compared with 
A

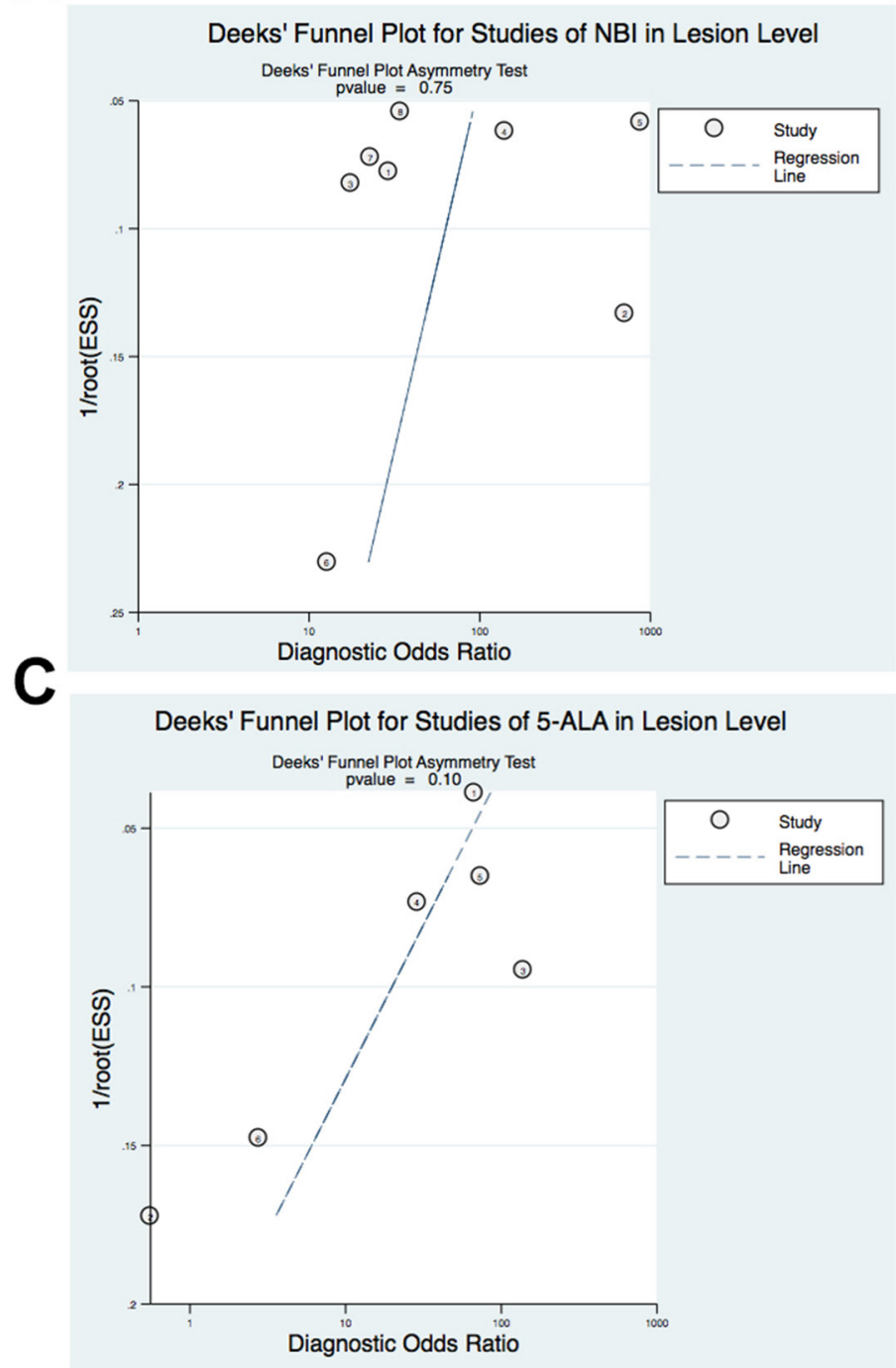

B

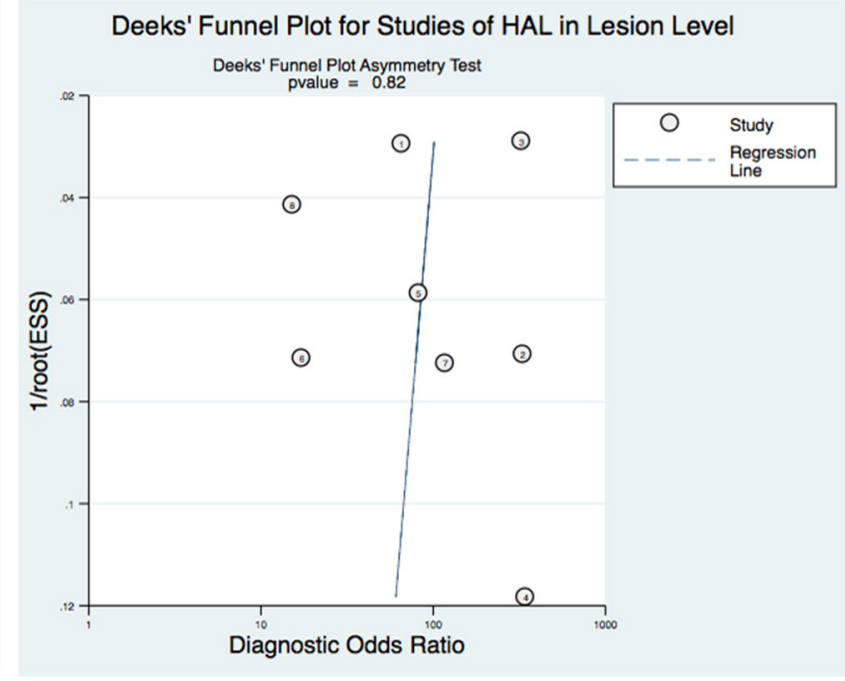

Figure 4 Deeks' funnel plot with asymmetry test for NBI (A), HAL (B) and 5-ALA (C) in lesion level. 5-ALA, 5-aminolevulinic acid; HAL, hexaminolevulinic acid; NBI, narrow band imaging.

WLC. ${ }^{11}{ }^{49}$ However, the application of these new imaging techniques has been limited by their increased false positives caused by intravesical instillation or inflammation, their technical complexity and increased cost. $^{13-16}$ It remains uncertain which technique could better improve the diagnostic accuracy of bladder cancer detection beyond the standard WLC. Virtually all the techniques assessed in this review were based on the reference standard of WLC, and new technique-assisted cystoscopy showed diagnostic superiority compared with conventional WLC. In this context, adoption of these strategies for practical bladder cancer diagnosis is essential. The results of the present study strongly suggested that new imaging-based technologies, in particular NBI, would be promising diagnostic interventions for bladder cancer detection in clinical practice.

In response to the latent disadvantages of WLC, PDD and NBI have been developed recently to improve the visualisation of bladder tumours. The diagnostic superiority of PDD or NBI over WLC for tumour detection has been demonstrated in several studies. ${ }^{115051}$ A meta-analysis comparing PDD with WLC found a $21 \%$ increase in tumour detection with PDD in the pooled estimates for both patients and biopsies. ${ }^{52}$ NBI, another optical enhancement technology, improves diagnostic accuracy by increasing the contrast of the superficial vasculature between the normal mucosa and tumour tissue. Previous studies reported significant improvement in the detection of bladder tumours using NBI cystoscopy compared with standard WLC. ${ }^{11} 13$ Our previous meta-analysis indicated that NBI identifies an additional $17 \%$ of patients and an additional $24 \%$ of tumours compared with WLC. ${ }^{53}$ However, these studies did not use standardised diagnostic accuracy definitions. Our diagnostic meta-analysis applied standard diagnostic accuracy definitions, and furthermore, the pooled estimates demonstrated that new technique assisted-cystoscopy had significant diagnostic superiority compared with conventional WLC, 
demonstrating the suboptimal performance of WLC in diagnosing NMIBC.

A study performed by Burger ${ }^{54}$ showed that HAL-assisted transurethral resection (TUR) significantly reduced the recurrence rate at 9-12 months compared with WLC-assisted TUR alone. In addition, Lee et $a b^{55}$ performed a meta-analysis evaluating oncological outcomes for WLC, PDD and NBI-assisted TUR, which showed that both PDD and NBI reduced the recurrence rate compared with WLC. However, the therapeutic effectiveness of new technique-assisted TUR, in terms of recurrence and progression, could not be demonstrated in the present review. Further therapeutic efficacy analysis is needed to identify promising interventions.

The strengths of our study include the stringent methodology used for searching and the study inclusion procedure, the standard definition of diagnostic performance and data extraction, the use of the strict diagnostic meta-analysis and the specific QUADAS-2 tool for RoB assessment. Moreover, the strict diagnostic meta-analysis and further subgroup analysis was applied to synthesise the diagnostic accuracy to obtain a reliable result. However, potential study limitations should be acknowledged. The lack of data on important clinical variables, such as grade and stage of disease, primary vs recurrent disease and intravesical instillation settings, might have introduced clinical heterogeneity and prevented further subgroup analyses. The predictive performance of recurrence or progression was not demonstrated in our study, which might decrease the reliability of diagnostic performance. We have attempted to minimise bias throughout the whole procedure, with rigorous search and selection criteria, standard data extraction and recalculation and subgroup analysis application, to evaluate the robustness of our findings.

In summary, this meta-analysis provided pooled diagnostic accuracy for NBI, HAL and 5-ALA techniques for patients with NMIBC in comparison with WLC as the reference standard. The results demonstrated that the diagnostic performances of NBI, HAL and 5-ALA were superior to that of WLC at the lesion level in diagnostic meta-analysis. The findings demonstrate the superior diagnostic performance of new imaging techniques in bladder detection compared with conventional WLC. These new imaging techniques are promising diagnostic interventions to improve clinical procedures in bladder cancer detection.

Acknowledgements We would like to thank Professor J X Zhang, Department of Medical Statistics and Epidemiology, School of Public Health, Sun Yat-Sen University, Guangzhou, China, for statistical advice and research comments.

Contributors CC conceptualised and designed the study, drafted the initial and final manuscript and provided funding support. HH contributed to data collection and extraction, data analysis and interpretation and drafted initial and final manuscript. YZ contributed to article screening, data collection and extraction, assessment of risk of bias and drafting manuscript. $\mathrm{HL}$ contributed to article screening, data collection and extraction and assessment of risk of bias. RS led and supervised statistical analysis and provided administrative support. TL and $\mathrm{JH}$ contributed to study conceptualization and design, supervised study implementation and critically reviewed the manuscript.
Funding This study was funded by the National Natural Science Foundation of China (Grant No. 81572514, 81472384, 81472381, 81402106, 81772719, 81772728, 91740119 and 91529301); Guangdong Medical Research Fund (A2018330); Science and Technology Program of Guangzhou (Grant No. 201604020156, 201604020177, 201707010116 and 201803010098); National Natural Science Foundation of Guangdong (Grant No. 2018A030313564, 2018B030311009, 2018A030310250, 2016A030313321, 2015A030311011 and 2015A030310122). Yixian Youth Project of Sun Yat-sen Memorial Hospital (YXQH201812).

Competing interests None declared.

Patient consent for publication Not required.

Provenance and peer review Not commissioned; externally peer reviewed.

Data availability statement Data are available in a public, open access repository. There are no data in this work. Data are available on reasonable request. Data may be obtained from a third party and are not publicly available. No data are available. All data relevant to the study are included in the article or uploaded as supplementary information.

Open access This is an open access article distributed in accordance with the Creative Commons Attribution Non Commercial (CC BY-NC 4.0) license, which permits others to distribute, remix, adapt, build upon this work non-commercially, and license their derivative works on different terms, provided the original work is properly cited, appropriate credit is given, any changes made indicated, and the use is non-commercial. See: http://creativecommons.org/licenses/by-nc/4.0/.

\section{REFERENCES}

1 Antoni S, Ferlay J, Soerjomataram I, et al. Bladder cancer incidence and mortality: a global overview and recent trends. Eur Urol 2017;71:96-108.

2 Chen C, He W, Huang J, et al. LNMAT1 promotes lymphatic metastasis of bladder cancer via CCL2 dependent macrophage recruitment. Nat Commun 2018;9:3826.

3 Schwaibold HE, Sivalingam S, May F, et al. The value of a second transurethral resection for T1 bladder cancer. BJU Int 2006;97:1199-201.

4 Sylvester RJ, van der Meijden APM, Oosterlinck W, et al. Predicting recurrence and progression in individual patients with stage TA T1 bladder cancer using EORTC risk tables: a combined analysis of 2596 patients from seven EORTC trials. Eur Urol 2006;49:466-77.

5 Grimbergen MCM, van Swol CFP, Jonges TGN, et al. Reduced specificity of 5-ALA induced fluorescence in photodynamic diagnosis of transitional cell carcinoma after previous intravesical therapy. Eur Urol 2003;44:51-6.

6 Daniltchenko DI, Riedl CR, Sachs MD, et al. Long-Term benefit of 5-aminolevulinic acid fluorescence assisted transurethral resection of superficial bladder cancer: 5 -year results of a prospective randomized study. J Urol 2005;174:2129-33.

7 Kriegmair M, Baumgartner R, Knüchel R, et al. Detection of early bladder cancer by 5-aminolevulinic acid induced porphyrin fluorescence. J Urol 1996;155:105-10.

8 Bryan RT, Shah ZH, Collins SI, et al. Narrow-Band imaging flexible cystoscopy: a new user's experience. J Endourol 2010;24:1339-43.

9 Naito S, Algaba F, Babjuk M, et al. The clinical research office of the endourological Society (CROES) multicentre randomised trial of narrow band Imaging-Assisted transurethral resection of bladder tumour (TURBT) versus conventional white light Imaging-Assisted TURBT in primary non-muscle-invasive bladder cancer patients: trial protocol and 1-year results. Eur Urol 2016;70:506-15.

10 Naselli A, Introini C, Timossi L, et al. A randomized prospective trial to assess the impact of transurethral resection in narrow band imaging modality on non-muscle-invasive bladder cancer recurrence. Eur Urol 2012;61:908-13.

11 Ye Z, Hu J, Song X, et al. A comparison of NBI and WLI cystoscopy in detecting non-muscle-invasive bladder cancer: a prospective, randomized and multi-center study. Sci Rep 2015;5:10905.

12 Xiong Y, Li J, Ma S, et al. A meta-analysis of narrow band imaging for the diagnosis and therapeutic outcome of non-muscle invasive bladder cancer. PLoS One 2017;12:e0170819.

13 Geavlete B, Multescu R, Georgescu D, et al. Narrow band imaging cystoscopy and bipolar plasma vaporization for large nonmuscleinvasive bladder tumors--results of a prospective, randomized comparison to the standard approach. Urology 2012;79:846-52.

14 Draga ROP, Grimbergen MCM, Kok ET, et al. Photodynamic diagnosis (5-aminolevulinic acid) of transitional cell carcinoma 
after Bacillus Calmette-Guérin immunotherapy and mitomycin C intravesical therapy. Eur Urol 2010;57:655-60.

15 Ray ER, Chatterton K, Khan MS, et al. Hexylaminolaevulinate fluorescence cystoscopy in patients previously treated with intravesical Bacille Calmette-Guérin. BJU Int 2010;105:789-94.

16 Daneshmand S, Schuckman AK, Bochner BH, et al. Hexaminolevulinate blue-light cystoscopy in non-muscleinvasive bladder cancer: review of the clinical evidence and consensus statement on appropriate use in the USA. Nat Rev Urol 2014:11:589-96.

17 Stroup DF, Berlin JA, Morton SC, et al. Meta-Analysis of observational studies in epidemiology: a proposal for reporting. meta-analysis of observational studies in epidemiology (moose) group. JAMA 2000;283:2008-12.

18 Bossuyt PMet al. Towards complete and accurate reporting of studies of diagnostic accuracy: the STARD initiative. BMJ 2003;326:41-4.

19 Moher D, Liberati A, Tetzlaff J, et al. Preferred reporting items for systematic reviews and meta-analyses: the PRISMA statement. Int $J$ Surg 2010;8:336-41.

20 Bossuyt PM, Reitsma JB, Bruns DE, et al. Stard 2015: an updated list of essential items for reporting diagnostic accuracy studies. BMJ 2015;351:h5527.

21 Whiting PF, Rutjes AWS, Westwood ME, et al. QUADAS-2: a revised tool for the quality assessment of diagnostic accuracy studies. Ann Intern Med 2011;155:529-36.

22 Ebell MH, Siwek J, Weiss BD, et al. Strength of recommendation taxonomy (SORT): a patient-centered approach to grading evidence in the medical literature. Am Fam Physician 2004;17:59-67.

23 Hanley JA, McNeil BJ. The meaning and use of the area under a receiver operating characteristic $(\mathrm{ROC})$ curve. Radiology 1982;143:29-36.

24 Song F, Khan KS, Dinnes J, et al. Asymmetric funnel plots and publication bias in meta-analyses of diagnostic accuracy. Int $J$ Epidemiol 2002;31:88-95.

25 Deeks JJ, Macaskill P, Irwig L. The performance of tests of publication bias and other sample size effects in systematic reviews of diagnostic test accuracy was assessed. $J$ Clin Epidemiol 2005;58:882-93.

26 Zhu Y-P, Shen Y-J, Ye D-W, et al. Narrow-Band imaging flexible cystoscopy in the detection of clinically unconfirmed positive urine cytology. Urol Int 2012;88:84-7.

27 Zaak D, Kriegmair M, Stepp H, et al. Endoscopic detection of transitional cell carcinoma with 5-aminolevulinic acid: results of 1012 fluorescence endoscopies. Urology 2001;57:690-4.

28 Tatsugami K, Kuroiwa K, Kamoto T, et al. Evaluation of narrow-band imaging as a complementary method for the detection of bladder cancer. J Endourol 2010;24:1807-11.

29 Song $\mathrm{PH}$, Cho S, Ko YH. Decision based on narrow band imaging cystoscopy without a referential normal standard rather increases unnecessary biopsy in detection of recurrent bladder urothelial carcinoma early after intravesical instillation. Cancer Res Treat 2016;48:273-80.

30 Shen Y-J, Zhu Y-P, Ye D-W, et al. Narrow-band imaging flexible cystoscopy in the detection of primary non-muscle invasive bladder cancer: a "second look" matters? Int Urol Nephrol 2012;44:451-7.

31 Shadpour P, Emami M, Haghdani S. A comparison of the progression and recurrence risk index in non-muscle-invasive bladder tumors detected by narrow-band imaging versus white light cystoscopy, based on the EORTC scoring system. Nephrourol Mon 2016;8:e33240

32 Schmidbauer J, Remzi M, Klatte T, et al. Fluorescence cystoscopy with high-resolution optical coherence tomography imaging as an adjunct reduces false-positive findings in the diagnosis of urothelial carcinoma of the bladder. Eur Urol 2009;56:914-9.

33 Riedl CR, Plas E, Pflüger H. Fluorescence detection of bladder tumors with 5-amino-levulinic acid. J Endourol 1999;13:755-9.

34 Palou J, Hernández C, Solsona E, et al. Effectiveness of hexaminolevulinate fluorescence cystoscopy for the diagnosis of non-muscle-invasive bladder cancer in daily clinical practice: a Spanish multicentre observational study. BJU Int 2015;116:37-43.

35 Lapini A, Minervini A, Masala A, et al. A comparison of

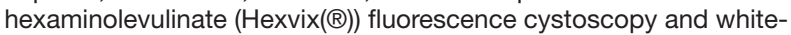

light cystoscopy for detection of bladder cancer: results of the HeRo observational study. Surg Endosc 2012;26:3634-41.

36 Kobatake K, Mita K, Ohara S, et al. Advantage of transurethral resection with narrow band imaging for non-muscle invasive bladder cancer. Oncol Lett 2015;10:1097-102.

37 Jichlinski P, Guillou L, Karlsen SJ, et al. Hexyl aminolevulinate fluorescence cystoscopy: new diagnostic tool for photodiagnosis of superficial bladder cancer--a multicenter study. $J$ Urol 2003;170:226-9.

38 Jeon SS, Kang I, Hong JH, et al. Diagnostic efficacy of fluorescence cystoscopy for detection of urothelial neoplasms. J Endourol 2001;15:753-9.

39 Herr HW, Donat SM. A comparison of white-light cystoscopy and narrow-band imaging cystoscopy to detect bladder tumour recurrences. BJU Int 2008:102:1111-4.

40 Geavlete B, Multescu R, Georgescu D, et al. Hexaminolevulinate fluorescence cystoscopy and transurethral resection of the bladder in noninvasive bladder tumors. J Endourol 2009;23:977-81.

41 Fradet Y, Grossman HB, Gomella L, et al. A comparison of hexaminolevulinate fluorescence cystoscopy and white light cystoscopy for the detection of carcinoma in situ in patients with bladder cancer: a phase III, multicenter study. J Urol 2007;178:68-73.

42 Filbeck T, Roessler W, Knuechel R, et al. Clinical results of the transurethreal resection and evaluation of superficial bladder carcinomas by means of fluorescence diagnosis after intravesical instillation of 5-aminolevulinic acid. J Endourol 1999;13:117-21.

43 Filbeck T, Pichlmeier U, Knuechel R, et al. Do patients profit from 5 -aminolevulinic acid-induced fluorescence diagnosis in transurethral resection of bladder carcinoma? Urology 2002;60:1025-8.

44 Ehsan A, Sommer F, Haupt G, et al. Significance of fluorescence cystoscopy for diagnosis of superficial bladder cancer after intravesical instillation of delta aminolevulinic acid. Urol Int 2001;67:298-304.

45 De Dominicis C, Liberti M, Perugia G, et al. Role of 5-aminolevulinic acid in the diagnosis and treatment of superficial bladder cancer: improvement in diagnostic sensitivity. Urology 2001;57:1059-62.

46 D'Hallewin MA, Vanherzeele H, Baert L. Fluorescence detection of flat transitional cell carcinoma after intravesical instillation of aminolevulinic acid. Am J Clin Oncol 1998;21:223-5.

47 Cauberg ECC, Kloen S, Visser M, et al. Narrow band imaging cystoscopy improves the detection of non-muscle-invasive bladder cancer. Urology 2010;76:658-63.

48 Burgués JP, Conde G, Oliva J, et al. [Hexaminolevulinate photodynamic diagnosis in non-muscle invasive bladder cancer: experience of the BLUE group]. Actas Urol Esp 2011;35:439-45.

49 Kausch I, Sommerauer M, Montorsi F, et al. Photodynamic diagnosis in non-muscle-invasive bladder cancer: a systematic review and cumulative analysis of prospective studies. Eur Urol 2010;57:595-606.

50 Babjuk M, Soukup V, Petrík R, et al. 5-Aminolaevulinic acid-induced fluorescence cystoscopy during transurethral resection reduces the risk of recurrence in stage $\mathrm{Ta} / \mathrm{T} 1$ bladder cancer. BJU Int 2005;96:798-802.

51 Gkritsios P, Hatzimouratidis K, Kazantzidis S, et al. Hexaminolevulinate-guided transurethral resection of non-muscleinvasive bladder cancer does not reduce the recurrence rates after a 2-year follow-up: a prospective randomized trial. Int Urol Nephrol 2014:46:927-33.

52 Mowatt G, N'Dow J, Vale L, et al. Photodynamic diagnosis of bladder cancer compared with white light cystoscopy: systematic review and meta-analysis. Int J Technol Assess Health Care 2011;27:3-10.

$53 \mathrm{Li} \mathrm{K}$, Lin T, Fan X, et al. Diagnosis of narrow-band imaging in nonmuscle-invasive bladder cancer: a systematic review and metaanalysis. Int J Urol 2013;20:602-9.

54 Burger M, Grossman HB, Droller M, et al. Photodynamic diagnosis of non-muscle-invasive bladder cancer with hexaminolevulinate cystoscopy: a meta-analysis of detection and recurrence based on RAW data. Eur Urol 2013;64:846-54.

55 Lee JY, Cho KS, Kang DH, et al. A network meta-analysis of therapeutic outcomes after new image technology-assisted transurethral resection for non-muscle invasive bladder cancer: 5-aminolaevulinic acid fluorescence vs hexylaminolevulinate fluorescence vs narrow band imaging. BMC Cancer 2015;15:566 